\title{
Simulation and experiment of substrate aluminum grain orientation dependent self-ordering in anodic porous alumina
}

\author{
Chuan Cheng, ${ }^{1, a)}$ K. Y. Ng, ${ }^{1}$ N. R. Aluru, ${ }^{2}$ and A. H. W. Ngan ${ }^{1}$ \\ ${ }^{1}$ Department of Mechanical Engineering, The University of Hong Kong, Pokfulam Road, Hong Kong, \\ People's Republic of China \\ ${ }^{2}$ Department of Mechanical Science and Engineering, Beckman Institute for Advanced Science and \\ Technology, University of Illinois at Urbana-Champaign, Urbana, Illinois 61801, USA
}

(Received 8 March 2013; accepted 6 May 2013; published online 22 May 2013)

\begin{abstract}
Recent experiments have indicated a strong influence of the substrate grain orientation on the self-ordering in anodic porous alumina. Anodic porous alumina with straight pore channels grown in a stable, self-ordered manner is formed on (001) oriented Al grain, while disordered porous pattern is formed on (101) oriented $\mathrm{Al}$ grain with tilted pore channels growing in an unstable manner. In this work, numerical simulation of the pore growth process is carried out to understand this phenomenon. The rate-determining step of the oxide growth is assumed to be the Cabrera-Mott barrier at the oxide/electrolyte $(\mathrm{o} / \mathrm{e})$ interface, while the substrate is assumed to determine the ratio $\beta$ between the ionization and oxidation reactions at the metal/oxide $(\mathrm{m} / \mathrm{o})$ interface. By numerically solving the electric field inside a growing porous alumina during anodization, the migration rates of the ions and hence the evolution of the o/e and m/o interfaces are computed. The simulated results show that pore growth is more stable when $\beta$ is higher. A higher $\beta$ corresponds to more $\mathrm{Al}$ ionized and migrating away from the $\mathrm{m} / \mathrm{o}$ interface rather than being oxidized, and hence a higher retained O:Al ratio in the oxide. Experimentally measured oxygen content in the self-ordered porous alumina on (001) $\mathrm{Al}$ is indeed found to be about 3\% higher than that in the disordered alumina on (101) $\mathrm{Al}$, in agreement with the theoretical prediction. The results, therefore, suggest that ionization on (001) Al substrate is relatively easier than on (101) $\mathrm{Al}$, and this leads to the more stable growth of the pore channels on (001) Al. (C 2013 AIP Publishing LLC.
\end{abstract}

[http://dx.doi.org/10.1063/1.4807295]

\section{INTRODUCTION}

Anodization of aluminum in an acidic or alkaline electrolyte can form a porous-type alumina with a quasihexagonal arrangement of the nanopore channels by selfassembly. ${ }^{1-5}$ The fabricated anodic porous alumina has recently been extensively utilized as templates for the synthesis of nano-structured materials by direct deposition or replication, for applications including optics, ${ }^{6}$ electronics, ${ }^{7}$ magnetic memories, ${ }^{8}$ and biodevices. ${ }^{9}$ The self-ordering quality of the pore arrangement in anodic porous alumina, which is an essential requirement for its application as templates, ${ }^{2,8}$ can be affected by various anodization conditions such as the electrolyte type and concentration, ${ }^{10}$ voltage, ${ }^{3}$ temperature, ${ }^{2}$ and time. ${ }^{11,12}$ Recently, $\mathrm{Ng}$ and Ngan ${ }^{5,13,14}$ reported that under the same anodization conditions, porous alumina with the best self-ordering quality is formed on (001) oriented $\mathrm{Al}$ grains, while the worst self-ordering is formed on (101) oriented $\mathrm{Al}$ grains, and other substrate orientations such as (111) give rise to intermediate pore ordering. Beck et al. ${ }^{15,16}$ also reported that (001) Al grain orientation is better than other orientations for self-ordered anodic porous alumina formation. They proposed that this was caused by an interfacial energy term of the driving force for the formation of the nanoporous alumina, i.e., the interfacial energy was reduced on (001) Al grains, increased on

${ }^{\text {a)} E l e c t r o n i c ~ m a i l: ~ c h u a n . c h e n g . r e s e a r c h @ g m a i l . c o m . ~}$
(111) grains, and could not decrease on (101) grains. ${ }^{15,16}$ According to this assumption, the rank of ordering quality should be $(001)>(101)>(111)$; yet, the experimentally observed rank was $(001)>(111)>(101) .{ }^{5}$ Most recently, Napolskii et al. ${ }^{17}$ reported that the in-plane orientation of the porous pattern in anodic porous alumina was determined by the crystallographic orientation of the $\mathrm{Al}$ substrate. As is similar to the proposal by Beck et al., ${ }^{15,16}$ the reason was assumed to be the minimization of surface energy which could cause the formation of an interface consisting of the most stable faces. ${ }^{17}$ Following this assumption, the predicted ultimate in-plane orientation ordering should be formed on (111) Al substrate, ${ }^{17}$ and again, this contradicts the experimental observation that pore ordering on (111) $\mathrm{Al}$ substrates is not better than on (001) substrates. ${ }^{5}$ Furthermore, the predicted shape of the bottoms of the pore channels at the metal/oxide (m/o) interface from the minimumsurface-energy assumption was facetted comprising piecewise flat crystallographic surfaces, ${ }^{15-17}$ but experimental observations (see Figs. 5(b) and 5(d)) show that the m/o interface has a scalloped shape comprising smooth and spherical domes. Thus, the mechanism behind the dependence of self-ordering in porous alumina on substrate grain orientation has not been understood. Considerations other than minimization of interfacial energy may be necessary.

In this paper, the substrate orientation effect on self-ordering in anodic porous alumina is investigated by numerical simulation using a previously established kinetics 
model. ${ }^{18}$ In this model, the electric field within the porous alumina during the anodization process assists oxide formation at the $\mathrm{m} / \mathrm{o}$ interface and oxide decomposition at the oxide/electrolyte (o/e) interface. Both $\mathrm{Al}^{3+}$ and $\mathrm{O}^{2-}$ ions migrate across these interfaces and the oxide barrier layer of the porous alumina according to the Cabrera-Mott equation in the high-electric-field theory. The effect of the substrate orientation is represented by the ratio $\beta$ of the ionization and oxidation reactions at the $\mathrm{m} / \mathrm{o}$ interface. The growth stability of the pore channels is investigated with respect to $\beta$ as a model parameter. Experimentally, since a higher value of $\beta$ corresponds to more $\mathrm{Al}$ ionization and migration away from $\mathrm{m} / \mathrm{o}$ interface rather than being oxidized and remaining in the oxide layer, the retained oxygen to aluminum ratio in the oxide is a reflection of the $\beta$ value. For this reason, the oxygen content in the oxide with different self-ordering is also experimentally investigated, in an attempt to rationalize with the theoretical predictions.

\section{SUBSTRATE GRAIN ORIENTATION DEPENDENT SELF-ORDERING}

Although the phenomenon of substrate grain orientation dependent self-ordering in anodic porous alumina has been reported elsewhere, ${ }^{5,13,14}$ the key experimental evidence reported here is obtained under different anodization conditions from previous work. ${ }^{5,13-17}$ Before anodization, pure polycrystalline $\mathrm{Al}$ foils $(99.99 \%)$ were annealed under vacuum $\left(\sim 10^{-5}\right.$ Torr $)$ at $500^{\circ} \mathrm{C}$ for $48 \mathrm{~h}$, followed by mechanical polishing with $1200,2400,4000$ grit $\mathrm{SiC}$ sandpapers and $6 \mu \mathrm{m}, 1 \mu \mathrm{m}$ diamond pastes in succession, and finally electropolishing in a mixture solution of $\mathrm{HClO}_{4}(60 \%$ wt.) and $\mathrm{C}_{2} \mathrm{H}_{5} \mathrm{OH}$ with $1: 4$ volume ratio under $20 \mathrm{~V}$ at about $-10^{\circ} \mathrm{C}$ for $2 \mathrm{~min}$. The anodization experiments were conducted in a large electrochemical cell $(2 \mathrm{l})$ in a constant temperature environment achieved by an electronic feed-back controlled water bath. The $\mathrm{Al}$ foils were mounted on a copper plate serving as the anode, while the cathode is an array of carbon rods placed $\sim 10 \mathrm{~cm}$ from the anode. The anodization condition for the experiments described in this section was $40 \mathrm{~V}$, $0.5 \mathrm{M} \mathrm{H}_{2} \mathrm{C}_{2} \mathrm{O}_{4}$, and $5^{\circ} \mathrm{C}$. Alumina formed under another condition of $40 \mathrm{~V}, 0.3 \mathrm{M} \mathrm{H}_{2} \mathrm{C}_{2} \mathrm{O}_{4}$, and $17^{\circ} \mathrm{C}$ is shown in Fig. 5 . Note that these two conditions are just examples, and the substrate orientation dependent self-ordering in anodic porous alumina is a general phenomenon under a wide range of anodization conditions; more evidence can be found in our recent paper. ${ }^{19}$ Even though the anodization conditions are different, as long as the ordering difference appears on different orientated substrate, the ordering rank is always $(001)>(111)>(101))^{5,19}$

After the 1 st step anodization for $18 \mathrm{~h}$, the anodic porous alumina formed on the substrate was selectively dissolved in a mixed solution of $\mathrm{H}_{2} \mathrm{CrO}_{4}, \mathrm{H}_{3} \mathrm{PO}_{4}$, and $\mathrm{H}_{2} \mathrm{O}$ with composition 1.8:6:92.2 by weight at $60^{\circ} \mathrm{C}$ for $3 \mathrm{~h}$. Then, the remaining $\mathrm{Al}$ substrate was marked by microscopically distinguishable markers by pen, and electron back-scattered diffraction (EBSD) was performed on the $\mathrm{Al}$ substrate in order to detect the crystallographic orientation of the $\mathrm{Al}$ grains, especially the locations of the grain boundaries between the (001) and
(101) grains. As shown in Fig. 1(a), white lines are the markers helping to locate the (101)/(001) Al grain boundaries. Fig. 1(b) shows pit patterns left on the Al substrate across a $(101) /(001)$ grain boundary in the framed region in Fig. 1(a). Due to the scalloped shape of the barrier layer in anodic porous alumina, each pore will leave a pit on the $\mathrm{Al}$ substrate, and so the pattern of the pits should directly reflect the arrangement of the pores at the end of the 1st step anodization. It is clear that the porous pattern on the right (001) Al grain is highly self-ordered into a quasi-hexagonal arrangement of sub-honeycomb zones of sizes 1 to $2 \mu \mathrm{m}$. On the contrary, the porous pattern on the left (101) Al grain is disordered with pits almost randomly arranged. Note that before anodization, the $\mathrm{Al}$ grains with different orientations were pre-treated in the same way, and the anodization conditions were also the same for different grains, thus the ordering difference of porous patterns is only due to the crystallographic orientations of the $\mathrm{Al}$ grains.

The 2nd step anodization was then conducted under the same conditions as in the 1st step for $14 \mathrm{~h}$. Scanning electron microscopy (SEM) was carried out in a LEO 1530 fieldemission microscope in order to observe the in-plane porous patterns, as shown in Fig. 1(c). To quantitatively evaluate the ordering of the porous pattern, the coordinates of the pore centers were captured by the IMAGEJ software. ${ }^{20}$ Then, these were statistically analyzed and plotted into a twodimensional (2-D) radial distribution function, defined as $\mathrm{RDF}=S_{\text {pattern }} /[2 \pi r \mathrm{~N}(d n(r) / d r)]$, where $S_{\text {pattern }}$ is the pattern's area, $r$ is the distance between the centers of any two pores in the pattern, $N$ is the total number of pore pairs, and $n(r)$ is the number of pore pairs in which the pores are separated by a distance $\leq r$. The RDF gives the probability density of finding a neighbor pore distanced $r$ away from any given pore in the pattern. In Fig. 1(d), the horizontal axis is normalized by $r / \mathrm{D}_{\text {int }}$, where $\mathrm{D}_{\text {int }}$ is the first peak position in RDF in the real length scale. It can be seen that the first 7 RDF peaks of the porous pattern formed on (001) $\mathrm{Al}$ almost coincide with those of the perfect hexagonal pattern (blue dashed lines in Fig. 1(d)), indicating short-range ordering up to the 7th nearest neighbors; on the contrary, the pattern formed on (101) Al does not resemble the perfect hexagonal arrangement in any way.

To observe the cross-section view of the anodic porous alumina (Fig. 1(c)) formed on the (101) and (001) Al substrate, transmission electron microscope (TEM) samples were cut perpendicularly across the grain boundary by focused ion beam (FIB) milling in a Quanta 200 3D dual beam FIB/SEM system operating at $30 \mathrm{kV}$ ion beam voltage, with the current varied from $7 \mathrm{nA}$ for initial coarse milling to $0.3 \mathrm{nA}$ for final fine milling. The TEM sample was observed in a Philips CM100 TEM operating at $80 \mathrm{kV}$. As shown in Fig. 1(e), for the in-plane disordered alumina grown on top of the (101) Al grain, pore channels are branched as indicated by short arrows in the figure, i.e., one pore channel may split into two or more, or terminate its growth within the oxide. On the other hand, as marked by long arrows in Fig. 1(f), for the in-plane self-ordered alumina formed on (001) Al grain, the pore channels mainly grow straight without tilting or branching. These observations here are in 

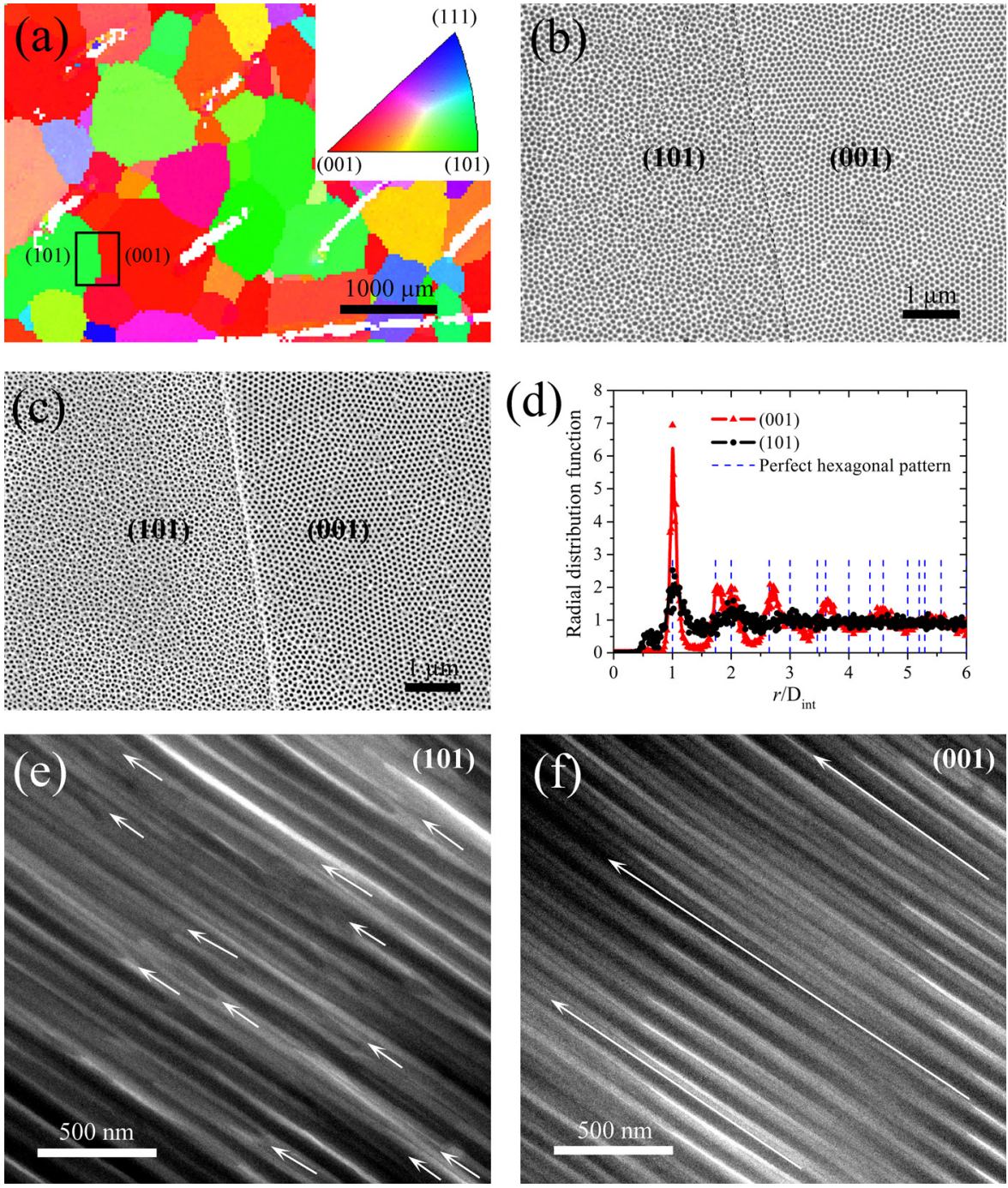

FIG. 1. (a) EBSD image of Al substrate after selectively dissolving the anodic porous alumina formed on top under the 1st step anodization $\left(40 \mathrm{~V}, 0.5 \mathrm{M} \mathrm{H}_{2} \mathrm{C}_{2} \mathrm{O}_{4}, 5^{\circ} \mathrm{C}, 18 \mathrm{~h}\right.$ ). (b) SEM topview of porous patterns on $\mathrm{Al}$ substrate captured around a (101)/(001) Al grain boundary. (c) SEM top-view of anodic porous alumina formed on the same location as (b) after the 2nd step anodization for $14 \mathrm{~h}$. (d) Radial distribution function of porous patterns in alumina formed on (101) and (001) Al grains. (e) and (f) TEM cross-sectional view of anodic porous alumina formed at the same location of (c) on (101) and (001) Al grains, respectively. accordance with previous findings. ${ }^{5}$ Thus, the in-plane selfordering difference of porous alumina is due to the growth stability of the pore channels during anodization.

\section{THEORETICAL MODEL}

\section{A. Reaction scheme and current densities}

A theoretical model is used to simulate the growth stability of porous alumina during anodization, the details of which can be found in our previous paper. ${ }^{18}$ Following Parkhutik and Shershulsky ${ }^{21}$ and Singh et al., ${ }^{22}$ when space charge within the oxide and double layer effects at the interfaces are neglected, the electric potential $\varphi$ within the anodic alumina is governed by the Laplace equation

$$
\nabla^{2} \varphi=0
$$

with boundary conditions $\varphi=0$ at the o/e interface, $\varphi=$ anodization voltage at the $\mathrm{m} / \mathrm{o}$ interface, and $\mathbf{n} \cdot \nabla \varphi=0$ on the left and right edges of alumina domain, where $\mathbf{n}$ is the outward normal unit vector of the two edges. The electric field is given as $\mathbf{E}=-\nabla \varphi$. During anodization, electrochemical reactions mainly take place at the $\mathrm{m} / \mathrm{o}$ and $\mathrm{o} / \mathrm{e}$ interfaces, ${ }^{23-26}$ where they can be assisted by high electric field. The reaction scheme is summarized in Fig. 2. At the m/ o interface, $\mathrm{Al}^{3+}$ ions are produced from the $\mathrm{Al}$ substrate by the ionization reaction

$$
\mathrm{Al}_{(\mathrm{m})} \rightarrow \mathrm{Al}_{(\mathrm{ox})}^{3+}+3 \mathrm{e}^{-}
$$

At the same time, the $\mathrm{Al}$ metal also undergoes the oxidation reaction

$$
2 \mathrm{Al}_{(\mathrm{m})}+3 \mathrm{O}_{(\text {ox })}^{2-} \rightarrow \mathrm{Al}_{2} \mathrm{O}_{3(\mathrm{ox})}+6 \mathrm{e}^{-}
$$

which will produce compression stress state at the m/o interface due to the volume expansion on oxidation. The $\mathrm{Al}^{3+}$ ions from Eq. (2) will migrate towards the o/e interface under the high electric field, and then be ejected into the electrolyte by ${ }^{27}$

$$
\mathrm{Al}_{(\mathrm{ox})}^{3+} \rightarrow \mathrm{Al}_{(\mathrm{aq})}^{3+} .
$$

The loss of $\mathrm{Al}^{3+}$ ions via Eqs. (2) and (4) will provide the necessary space to help relieve the compressive stress in the newly formed oxide at the $\mathrm{m} / \mathrm{o}$ interface due to the volume expansion $\left(\mathrm{Al} \rightarrow \mathrm{Al}_{2} \mathrm{O}_{3}\right.$ ) by Eq. (3). ${ }^{28}$ The needed $\mathrm{O}^{2-}$ ions in Eq. (3) come from the o/e interface by water decomposition at o/e interface ${ }^{27}$ 
Reactions at o/e interface:

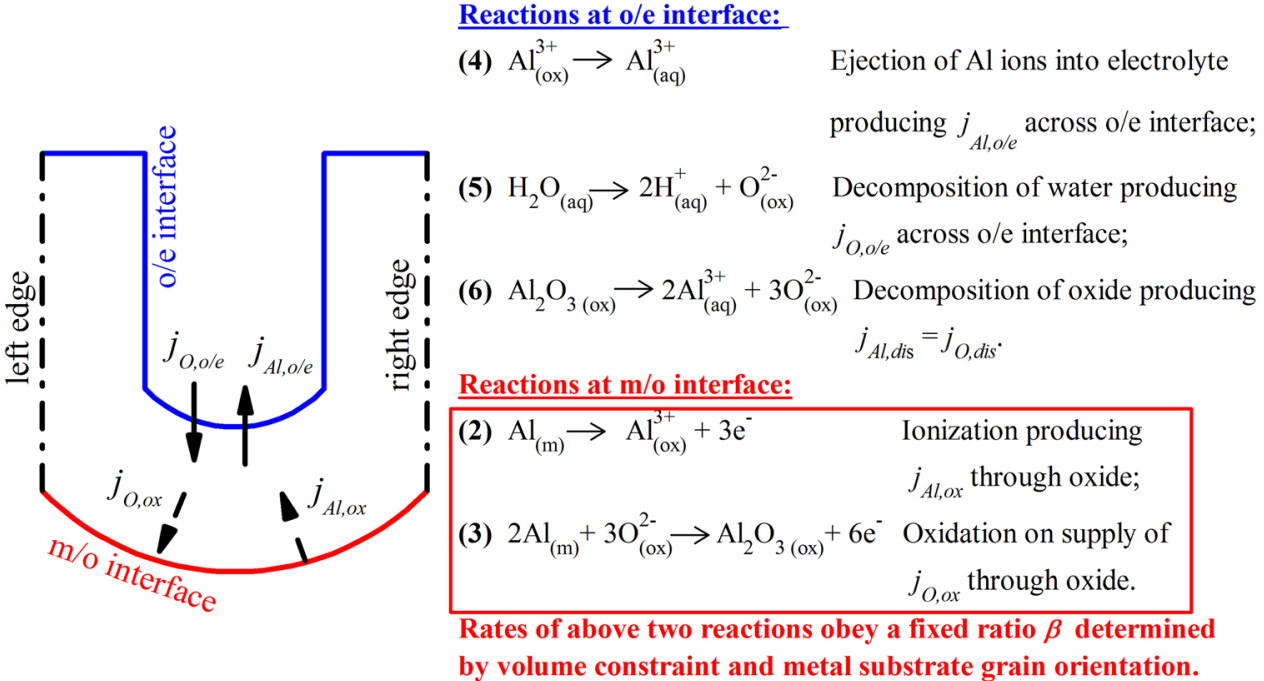

FIG. 2. The reaction scheme for the pore channel growth in anodic porous alumina.

\section{Rate-determining currents across potential barriers at o/e interface:}

\begin{tabular}{|c|c|c|c|c|c|}
\hline $\begin{array}{l}j_{O, o / e}= \\
\text { (5) }\end{array}$ & $\begin{array}{c}j_{O, o x} \\
\text { (3) }\end{array}$ & $-\begin{array}{c}j_{O, d i s} \\
(\mathbf{6})\end{array}$ & $\begin{array}{c}j_{A l, o / e} \\
\text { (4) }\end{array}$ & $\begin{array}{c}=j_{A l, o x}+ \\
\text { (2) }\end{array}$ & $\begin{array}{l}j_{A l, d i s} \\
\text { (6) }\end{array}$ \\
\hline
\end{tabular}

This drives $\mathrm{m} / \mathrm{o}$ interface movement These drive o/e interface movement. following Faraday's Law.

$$
\mathrm{H}_{2} \mathrm{O}_{(\mathrm{aq})} \rightarrow 2 \mathrm{H}_{(\mathrm{aq})}^{+}+\mathrm{O}_{(\mathrm{ox})}^{2-},
$$

and also by field-assisted alumina decomposition ${ }^{25}$

$$
\mathrm{Al}_{2} \mathrm{O}_{3(\mathrm{ox})} \rightarrow 2 \mathrm{Al}_{(\mathrm{aq})}^{3+}+3 \mathrm{O}_{(\mathrm{ox})}^{2-} .
$$

In Eq. (6), the product $\mathrm{O}^{2-}$ will not form water with $\mathrm{H}^{+}$in the electrolyte but migrate across the oxide layer to the m/o interface, because negligible loss of oxygen from alumina was detected experimentally, ${ }^{23,25,27}$ while the product $\mathrm{Al}^{3+}$ will also eject into the electrolyte by Eq. (4).

From the above analysis, the ejected $\mathrm{Al}^{3+}$ ions into the electrolyte come from two parts. One part is produced at the m/o interface via Eq. (2) and then migrates across the oxide to the electrolyte, the current density of which is denoted as $\mathbf{j}_{\text {Al,ox }}$, where "ox" means migration across the oxide, and the values of $\mathbf{j}_{A l, o x}$ at the o/e and $\mathrm{m} / \mathrm{o}$ interfaces are denoted as $\left.\mathbf{j}_{A l, o x}\right|_{o / e}$ and $\left.\mathbf{j}_{A l, o x}\right|_{m / o}$, respectively. The other part is produced by oxide decomposition at the o/e interface via Eq. (6), with a current density denoted as $\mathbf{j}_{A l \text {,dis }}$, which is equal to the current density of $\mathrm{O}^{2-}\left(\mathbf{j}_{O \text {,dis }}\right)$ produced from the same reaction, i.e.,

$$
\mathbf{j}_{A l, d i s}=\mathbf{j}_{O, d i s} .
$$

Thus, the total current density of $\mathrm{Al}^{3+}$ at the o/e interface is

$$
\mathbf{j}_{A l, o / e}=\left.\mathbf{j}_{A l, o x}\right|_{o / e}+\mathbf{j}_{A l, d i s} \text {. }
$$

The migration of $\mathrm{Al}^{3+}$ ions across the o/e interface is governed by the Cabrera-Mott equation in the high electric field theory 24,29

$$
\mathbf{j}_{A l, o / e}=n_{A l} A_{A l} \exp \left(k_{A l} E_{o / e}\right) \hat{\mathbf{E}}_{o / e},
$$

where $A_{A l}=C_{H^{+}}^{\eta} q_{A l} \nu_{A l} \exp \left(-W_{A l} / k T\right)$ and $k_{A l}=\alpha_{A l} q_{A l} a_{A l} /$ $k T, n_{A l}$ is the density of mobile $\mathrm{Al}^{3+}$ ions, $C_{H^{+}}$is the $\mathrm{H}^{+}$ concentration, $\eta=1,{ }^{21,22} q_{A l}$ is the charge of one $\mathrm{Al}^{3+}, \nu_{A l}$ is the vibration frequency of $\mathrm{Al}^{3+}, W_{A l}$ is the potential barrier without electric field, $\alpha_{A l}$ is a transfer coefficient related to the symmetry of the potential barrier, $a_{A l}$ is the jump distance of $\mathrm{Al}^{3+}, k$ is the Boltzmann constant, $T$ is the absolute temperature, and $\hat{\mathbf{E}}_{o / e}=\mathbf{E}_{o / e} / E_{\mathrm{o} / \mathrm{e}}$ is the unit vector of the electric field at the o/e interface.

Similarly, the current density of $\mathrm{O}^{2-}$ ions at the o/e interface also comes from two parts. One part is from water decomposition at the o/e interface by Eq. (5), the current density of which is denoted as $\mathbf{j}_{O, o / e}$. The other part is from oxide decomposition by Eq. (6), and the current density of which is $\mathbf{j}_{\text {o,dis }}\left(=\mathbf{j}_{\text {Al,dis }}\right)$. Thus, the total current density of $\mathrm{O}^{2-}$ ions at the o/e interface is

$$
\left.\mathbf{j}_{O, o x}\right|_{o / e}=\mathbf{j}_{O, o / e}+\mathbf{j}_{O, d i s} .
$$

Because the $\mathrm{O}^{2-}$ ions produced from oxide decomposition (jo,dis) will not lose into the electrolyte but migrate towards the $\mathrm{m} / \mathrm{o}$ interface to form new oxide, ${ }^{23}$ thus, only those $\mathrm{O}^{2-}$ ions coming from water decomposition (with current density $\mathbf{j}_{\text {o,o/e }}$ ) need to jump across the potential barrier at the o/e interface, and this current density also follows the CabreraMott equation, ${ }^{24,29}$ which is

$$
\mathbf{j}_{O, o / e}=n_{O} A_{O} \exp \left(k_{O} E_{o / e}\right) \hat{\mathbf{E}}_{o / e},
$$

where $A_{O}=q_{O} \nu_{O} \exp \left(-W_{O} / k T\right)$ and $k_{O}=\alpha_{O} q_{O} a_{O} / k T$, and the parameters in these expressions have similar meanings as in Eq. (9) albeit now for $\mathrm{O}^{2-}$ ions.

\section{B. Rate-determining step and role of the metal substrate}

Next, the rate-determining step of the pore-growth process and the role of the $\mathrm{Al}$ metal substrate need to be 
established. Recent experiments have revealed that an increase in the electrolyte concentration can influence the anodization process significantly, such as the pore diameter, ${ }^{30}$ the current density, ${ }^{31}$ and the oxide growth rate. ${ }^{32,33}$ Since the electrolyte makes contact with the oxide at the o/e interface, these profound effects of the electrolyte can only be consequences of the changes of the reactions at the o/e interface, rather than those at the $\mathrm{m} / \mathrm{o}$ interface pertinent to the $\mathrm{Al}$ substrate. For this reason, the rate-determining step for the oxide evolution is assumed to be the Cabrera-Mott barrier at the o/e interface in Eqs. (9) and (11). ${ }^{18}$

On the other hand, the experimental results in Sec. II above clearly indicate a strong effect of the substrate orientation on the stability of the oxide growth, and so the specificity of the $\mathrm{Al}$ substrate orientation at the $\mathrm{m} / \mathrm{o}$ interface needs to be represented in the model. Since the barrier at the o/e interface is rate determining, that at the $\mathrm{m} / \mathrm{o}$ would be unimportant and, therefore, cannot represent the specificity of the substrate orientation. However, the ionization and oxidation reactions, Eqs. (2) and (3), at the $\mathrm{m} / \mathrm{o}$ interface should depend on the $\mathrm{Al}$ substrate orientation. Therefore, we assume here that the specificity of the $\mathrm{Al}$ substrate orientation is represented by the ratio $\beta$ of the rates of Eqs. (2) and (3) at the $\mathrm{m} / \mathrm{o}$ interface. As said above, the ionization reaction Eq. (2) produces ion-current density $\left.\mathbf{j}_{A l, o x}\right|_{\text {m/o }}$, and the oxidation reaction Eq. (3) produces $\left.\mathbf{j}_{o, o x}\right|_{m / o}$, and so $\beta$ is defined as

$$
\beta=\frac{\left.j_{A l, o x}\right|_{m / o}}{\left.j_{O, o x}\right|_{m / o}},
$$

where $j$ is the current density magnitude corresponding to the vector $\mathbf{j}$, and as mentioned before, "ox" also means migration through the oxide, and " $\mathrm{I} / \mathrm{e}_{\mathrm{e}}$ " and " $\mathrm{I}_{\mathrm{m} / \mathrm{o}}$ " represent values at the $\mathrm{o} / \mathrm{e}$ and $\mathrm{m} / \mathrm{o}$ interfaces, respectively. The assumption here is that different orientations of the $\mathrm{Al}$ substrate give rise to different $\beta$ values.

However, by considering continuity of the steady-state ion current density $\mathbf{j}$ within the oxide $\nabla \cdot \mathbf{j}=0$ and Eq. (1), it can be shown that ${ }^{18}$

$$
\frac{j_{o / e}}{j_{m / o}}=\frac{E_{o / e}}{E_{m / o}}
$$

where the subscript "o/e" represents the corresponding value at a point on o/e interface, and " $\mathrm{m} / \mathrm{o}$ " represents the corresponding value at another point on $\mathrm{m} / \mathrm{o}$ interface, but the two points were connected by the same electric field line. ${ }^{18}$ Then, from Eqs. (12) and (13),

$$
\beta=\frac{\left.j_{A l, o x}\right|_{m / o}}{\left.j_{O, o x}\right|_{m / o}}=\frac{\left.j_{A l, o x}\right|_{o / e}}{\left.j_{O, o x}\right|_{o / e}} .
$$

Equation (14), therefore, indicates that if the current densities $\mathbf{j}_{A l, o x}$ and $\mathbf{j}_{O, o x}$ obey a given ratio $\beta$ at the $\mathrm{m} / \mathrm{o}$ interface, then the same ratio is maintained at the o/e interface, and in fact at any equipotential surface within the oxide. ${ }^{18}$ Thus, although the ratio $\beta$ is initially defined for the current densities at the $\mathrm{m} / \mathrm{o}$ interface to represent the specificity of the Al substrate as in Eq. (12), the same ratio is obeyed throughout the whole oxide layer, due to the continuity equation in Eq. (13). In experiments, under a certain anodization condition, the transport numbers of both ions were indeed found to be fixed, ${ }^{25}$ and this corresponds very well to the assumption that $\beta$ is a fixed value for a given $\mathrm{Al}$ substrate orientation.

\section{Movement velocities of interfaces}

Having now established that the rate-determining step is the Cabrera-Mott barrier at the o/e interface and yet the $\mathrm{Al}$ substrate determines a given ratio $\beta$ of the current densities of the $\mathrm{Al}^{3+}$ and $\mathrm{O}^{2-}$ ions throughout the oxide, the next step is to cast the movements of the o/e and $\mathrm{m} / \mathrm{o}$ interfaces in terms of the Cabrera-Mott barrier at the o/e interface as well as the parameter $\beta$. From Eqs. (7), (8), (10), and (14), and noting that $\left.\mathbf{j}_{\mathrm{Al}, o x}\right|_{\mathrm{o} / \mathrm{e}},\left.\mathbf{j}_{O, o x}\right|_{o / e}, \mathbf{j}_{A l, o / e}, \mathbf{j}_{O \text {,ole }}, \mathbf{j}_{A l \text {,dis }}$, and $\mathbf{j}_{\text {o,dis }}$ have the same direction $\hat{\mathbf{E}}_{o / e}$ at a given point on o/e interface,

$$
\mathbf{j}_{A l, d i s}=\frac{j_{A l, o / e}-\beta j_{O, o / e}}{1+\beta} \hat{\mathbf{E}}_{o / e}
$$

From Faraday's law, ${ }^{34}$ the moving velocity $\mathbf{v}$ of the oxide thickness $D=V / A$ at a given point at the interface is proportional to the current density as $\mathbf{v}=-(M \mathbf{j}) /(z F \rho)$, where $V$ is the volume of alumina oxide, $A$ is the area of oxide surface, $M$ is the molecular weight of oxide $\mathrm{Al}_{x} \mathrm{O}_{y}, z=x y, \rho$ is the oxide density, $\mathbf{j}$ is the current density corresponding to the reaction, and $F$ is Faraday's constant. More specifically, at the o/e interface, the interface movement velocity is $\mathbf{v}_{\text {ole }}=-\mathbf{j}_{A l, d i s} M / z F \rho$, and substituting in Eq. (15), and replacing $\mathbf{j}_{A l, \mathrm{o} / \mathrm{e}}$ and $\mathbf{j}_{O, \mathrm{o} / \mathrm{e}}$ by Eqs. (9) and (11), respectively, we obtain

$$
\begin{aligned}
\boldsymbol{v}_{o / e}= & -\frac{M}{z F \rho(1+\beta)}\left[n_{A l} A_{A l} \exp \left(k_{A l} E_{o / e}\right)\right. \\
& \left.-\beta n_{O} A_{O} \exp \left(k_{O} E_{o / e}\right)\right] \hat{\mathbf{E}}_{o / e} .
\end{aligned}
$$

Similarly, the $\mathrm{m} / \mathrm{o}$ interface movement velocity is $\mathbf{v}_{\mathrm{m} / \mathrm{o}}$ $=-\left.\mathbf{j}_{O, o x}\right|_{m / o} M / z F \rho$, and from Eqs. (7), (9), (10), (11), (13), and (15), this is given as

$$
\begin{aligned}
\mathbf{v}_{m / o}= & -\frac{M}{z F \rho(1+\beta)} \frac{E_{m / o}}{E_{o / e}}\left[n_{A l} A_{A l} \exp \left(k_{A l} E_{o / e}\right)\right. \\
& \left.+n_{O} A_{O} \exp \left(k_{O} E_{o / e}\right)\right] \hat{\mathbf{E}}_{m / o} .
\end{aligned}
$$

Here, $n_{A l}$ and $n_{O}$ are mobile ion densities at the o/e interface, which are found experimentally to depend exponentially on the electric field intensity. ${ }^{24}$ Thus, the following equation is used to represent the dependence for both ion species (for $\mathrm{O}^{2-}$ ions, the subscript $A l$ is replaced with $O$ )

$$
n_{A l}=n_{A l}^{0} \exp \left[\ln (\lambda)-\ln (\lambda) \frac{E_{o / e}}{E_{\text {cutoff }}}\right],
$$

where $n_{A l}^{0}$ is the number of $\mathrm{Al}^{3+}$ ions when all of them are mobile, and $\lambda=0.2{ }^{18}$ As observed in experiments, ${ }^{35}$ at the o/e interface, a double logarithmic plot of the $\mathrm{O}^{2-}$ ions current density $j_{O, o / e}$ versus the $\mathrm{Al}^{3+}$ ions current density $j_{A l, o / e}$ yielded 
straight lines corresponding to $\left(\partial \ln j_{O, o / e} / \partial \ln j_{A l, o / e}\right)_{p H}$ $=1.38( \pm 0.14)$, where the slope 1.38 is rather independent of the $\mathrm{pH}$ from 0 to 11 . Thus, from this relation and Eqs. (9) and (11) we set $k_{O} / k_{A l}=1.5$ in our simulations. $k_{O}$ was set (e.g., $3.8 \mathrm{~nm} \mathrm{~V}^{-1}$ ) to be the same order of magnitude as reported. ${ }^{21}$ Due to the contamination of the oxide by electrolyte ions, the oxide density $\rho$ may vary from place to place. At present, it is not clear how $\rho$ varies within the oxide volume and along each interfaces, and for simplicity's sake a constant value of $\rho=3.118 \mathrm{~g} \mathrm{~cm}^{-3}$ is used, which is agreeable to experiments. ${ }^{24}$ Variable oxide density along each interface can be adopted easily in the present model when the exact relation becomes clear in the future. Furthermore, the exact values of $n_{A l}^{0}, n_{O}^{0}$, $A_{A l}$, and $A_{O}$ for porous-type anodic alumina are hard to be measured in experiments. In order to reduce the complexity of Eqs. (16) and (17), we set

$$
\begin{gathered}
B_{A l}=n_{A l}^{0} A_{A l}=n_{A l}^{0} C_{H^{+}}^{\eta} q_{A l} \nu_{A l} \exp \left(-W_{A l} / k T\right), \\
B_{O}=n_{O}^{0} A_{O}=n_{O}^{0} q_{O} \nu_{O} \exp \left(-W_{O} / k T\right),
\end{gathered}
$$

as constants under a certain anodization condition. The values of $B_{A l}$ and $B_{O}$ are estimated based on reported values ${ }^{24}$ for each of the parameters involved in Eqs. (19) and (20) to produce oxide growth rates on the order of $1 \mathrm{~nm} \mathrm{~s}^{-1}$ at the pore base, which is a common experimental value under mild anodization conditions. ${ }^{2,3}$ For instance, a typical value of $B_{A l}=1 \mathrm{~A} \mathrm{~m}^{-2}$ can be achieved by setting the charge density $n_{A l}^{0} q_{\mathrm{Al}}=1800 \mathrm{C} \mathrm{cm}^{-3}$, vibration frequency $\nu=10^{12} \mathrm{~s}^{-1}$, temperature $T=275 \mathrm{~K}, \mathrm{pH}=1, \eta=1$, and potential barrier $W_{A l}=1.105 \mathrm{eV}$. These quantities are physically reasonable. ${ }^{24}$

\section{SIMULATION RESULTS}

Numerical implementation of the model discussed in Sec. III was realized based on the finite element method. A computer code was developed from the MATLAB PDE toolbox. ${ }^{36}$ Simulation of porous alumina growth starts from a pre-textured 2-D cross-section configuration of alumina at anodization time $\mathrm{t}=0 \mathrm{~s}$, as shown in Fig. 3(a). Two identical initial pores with diameter $20 \mathrm{~nm}$ and interpore distance $100 \mathrm{~nm}$ pre-exist on the surface of alumina, the width of which is $200 \mathrm{~nm}$ and thickness is $50 \mathrm{~nm}$. Under the same initial configuration but different values of the parameter $\beta$, the pore channels can grow into an unstable configuration under $\beta=0.4$ (Fig. 3(a)), and a stable configuration under $\beta=0.5$ (Fig. 3(b)), with anodization time increasing. In the unstable pore channel development in Fig. 3(a), pore termination and splitting are observed, while in the stable development in Fig. 3(b), pore channels grow straight downward. These two simulated configurations resemble very well the TEM crosssectional views in Figs. 1(e) and 1(f). For example, the scallop shaped barrier layer at the pores' bottom, which previously does not exist in the initial simulation configuration at $\mathrm{t}=0 \mathrm{~s}$, forms as anodization time increases, and the simulated barrier layer thickness of about $40 \mathrm{~nm}$ matches the experimental value very well (e.g., Figs. 5(b) and 5(d)). In Fig. 3(c), simulations are conducted under various values of $\beta$ and $B_{A l}$, while other parameters are the same. After the
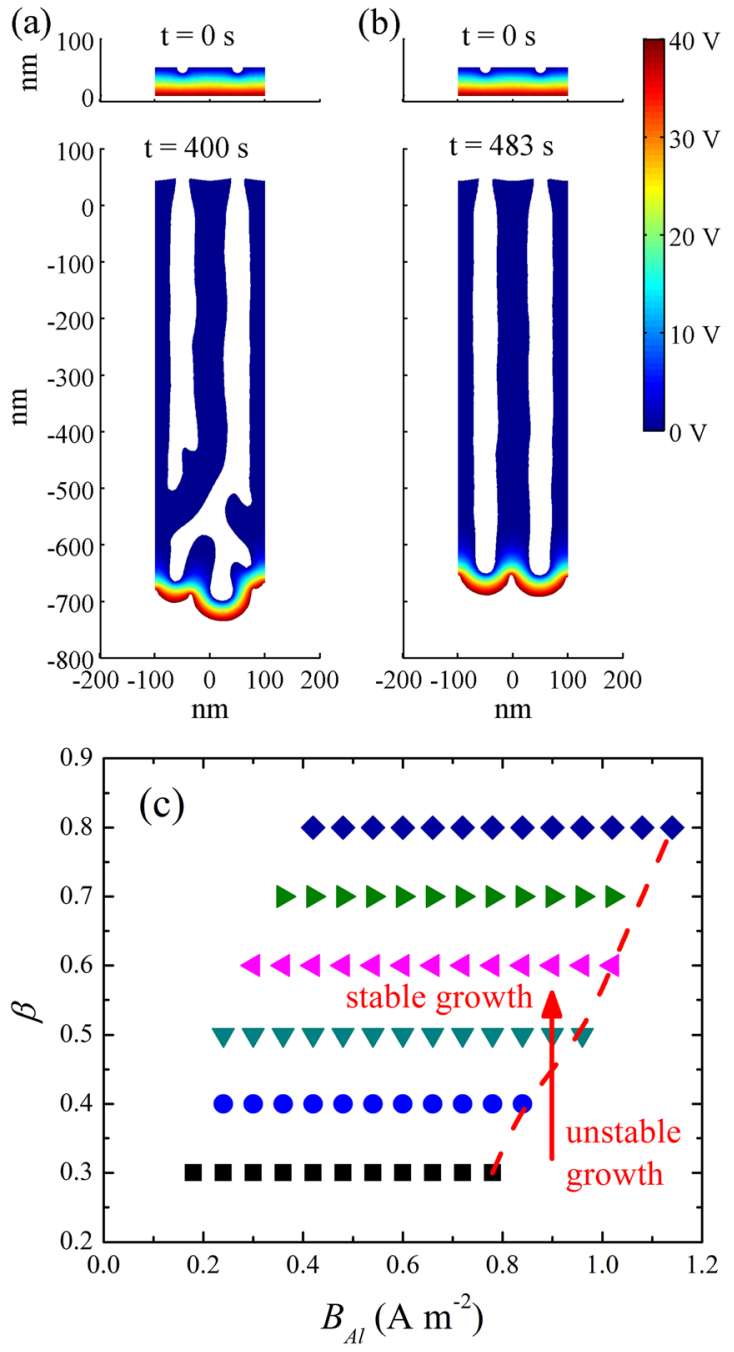

FIG. 3. (a) and (b) Simulation of pore channel growth in anodic porous alumina starting from the same pre-textured configuration $(\mathrm{t}=0 \mathrm{~s})$ with $\beta=0.4$ and 0.5 , for $\mathrm{t}=400$ and $483 \mathrm{~s}$ anodization time, respectively, while other simulation parameters are the same $\left(40 \mathrm{~V}, k_{O} / k_{A l}=1.5, k_{O}=3.8 \mathrm{~nm} \mathrm{~V}^{-1}\right.$, $B_{A l}=0.9 \mathrm{~A} \mathrm{~m}^{-2}$, and $B_{O}=0.072 \mathrm{~A} \mathrm{~m}^{-2}$ ). (c) Map of $\beta$ and $B_{A l}$ conditions for unstable and stable pore channel growth to occur.

same $300 \mathrm{~s}$ anodization time, only those parameter values which can result in stable pore channel development are plotted in Fig. 3(c) as a phase diagram. It can be seen that a boundary (the red dashed line) exists between unstable and stable pore channel development. With $\beta$ increasing along the vertical arrow direction in Fig. 3(c), the pore development can transform from unstable to stable, and Figs. 3(a) and 3(b) are only typical examples for such transition.

With the same pre-textured initial configuration as in Fig. 3, but different parameters of $k_{O}=4.2 \mathrm{~nm} \mathrm{~V}^{-1}$ and $B_{A l}=1.08 \mathrm{~A} \mathrm{~m}^{-2}$, simulated pore channel growth patterns with $\beta$ increasing from 0.3 to 0.4 are plotted in Figs. 4(a) and 4 (b), respectively. At $\beta=0.3$, after $210 \mathrm{~s}$ of anodization time, the pore channels develop into a very unstable configuration as shown in Fig. 4(a), where the right pore terminates its growth, while the left pore tilts and splits into two multiple channels forming a dendritic pattern. However, at a higher $\beta=0.4$ as shown in Fig. 4(b), the pore channels are straight without termination or splitting. The phase diagram 
(a)

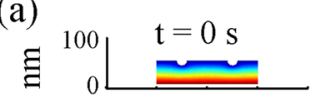

(b)
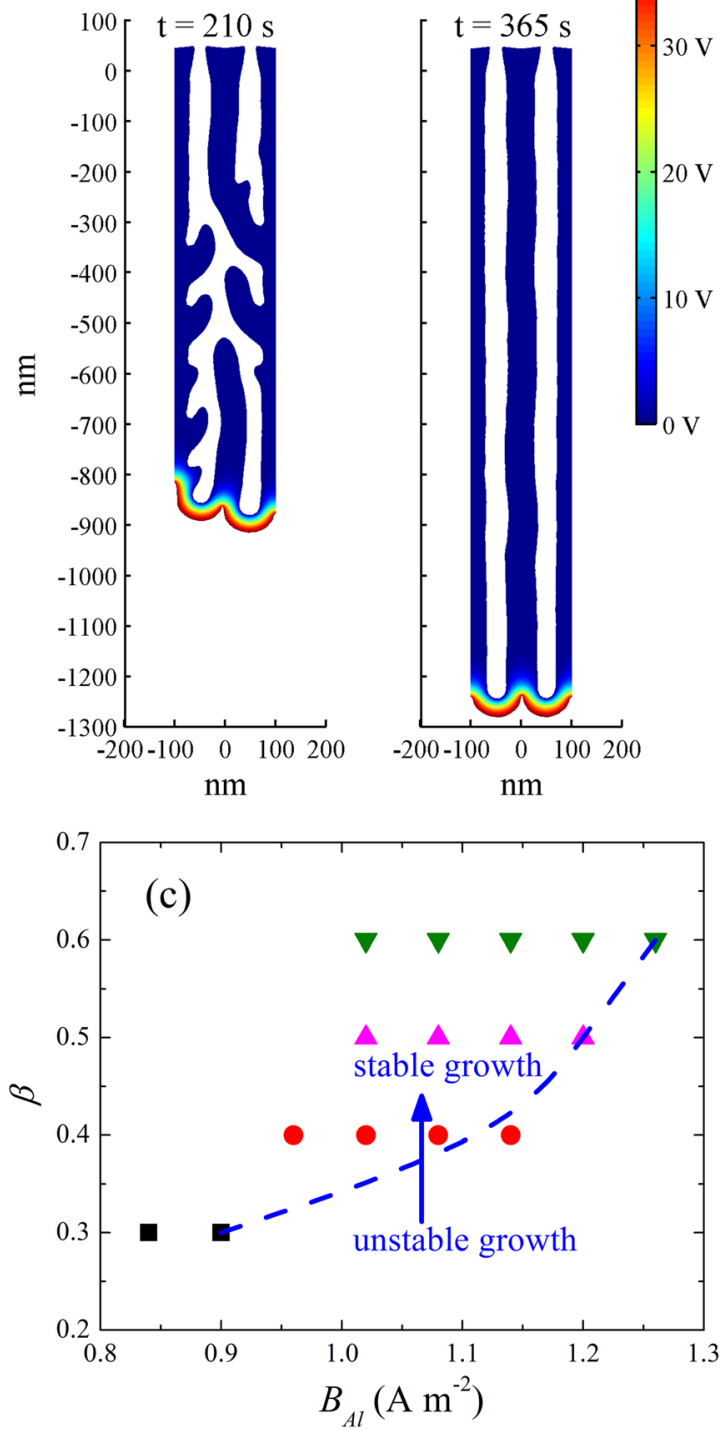

FIG. 4. (a) and (b) Simulation of pore channel growth starting from the same pre-textured configuration $(\mathrm{t}=0 \mathrm{~s}$ ) with $\beta=0.3$ and 0.4 , for $\mathrm{t}=210$ and $365 \mathrm{~s}$ anodization time, respectively, while other simulation parameters are the same $\left(40 \mathrm{~V}, k_{O} / k_{A l}=1.5, k_{O}=4.2 \mathrm{~nm} \mathrm{~V}^{-1}, B_{A l}=1.08 \mathrm{~A} \mathrm{~m}^{-2}\right.$, and $B_{O}=0.072 \mathrm{~A} \mathrm{~m}^{-2}$ ). (c) Map of $\beta$ and $B_{A l}$ conditions for unstable and stable pore channel growth to occur.

in Fig. 4(c) shows that, compared with Fig. 3(c), the stable region for pore channel development is rather narrow, which means that the unstable-to-stable transformation is also dependent on the simulation parameter $k_{O}$, but the transformation from unstable to stable also happens as $\beta$ increases, as shown by the blue arrow in Fig. 4(c). Figs. 4(a) and 4(b) actually represent a typical example of this transformation.

\section{DISCUSSION}

\section{A. Physical meaning and effects of $\beta$}

The simulated results in Figs. 3 and 4 indicate a general trend of unstable-to-stable transformation on increasing $\beta$, and so in this section the physical meaning and effects of this model parameter are further exploited. From Eq. (17), the movement velocity of the $\mathrm{m} / \mathrm{o}$ interface also yields the volume of oxide transformed from metal per unit interface area per unit time, i.e.,

$$
v_{m / o}=\left.j_{O, o x}\right|_{m / o} \frac{V_{o x}}{z F},
$$

where $V_{o x}$ is the molecular volume of oxide $\mathrm{Al}_{x} \mathrm{O}_{y}$ and $z=x y$. Let the Pilling-Bedworth ratio ${ }^{28,37}$ due to volume expansion $\left(\mathrm{Al} \rightarrow \mathrm{Al}_{x} \mathrm{O}_{y}\right)$ be $\left(1+\varepsilon_{V}\right)$, where $\varepsilon_{V}$ means the ratio of the expanded volume compared with the previous volume of Al. Then, the expanded volume in the newly formed oxide per unit $\mathrm{m} / \mathrm{o}$ interface area per unit time is

$$
v_{\exp a n}=\left.j_{O, o x}\right|_{m / o} \frac{V_{o x}}{z F} \frac{\varepsilon_{V}}{1+\varepsilon_{V}} .
$$

However, the loss of $\mathrm{Al}$ produced by Eq. (2) at the m/o interface, migrating towards the o/e interface and then ejected into electrolyte by Eq. (4), will provide some spacing at the $\mathrm{m} / \mathrm{o}$ interface for the newly formed oxide. This spacing for $\mathrm{Al}$ lost per unit $\mathrm{m} / \mathrm{o}$ interface area per unit time equals

$$
v_{\text {space }}=\left.j_{A l, o x}\right|_{m / o} \frac{V_{A l}}{y F},
$$

where $\left.j_{A l, o x}\right|_{m / o}$ is the magnitude of the current density of $\mathrm{Al}^{3+}$ ions at the $\mathrm{m} / \mathrm{o}$ interface, which will migrate to the o/e interface to be lost there, $V_{A l}$ is the molecular volume of $\mathrm{Al}$, and $y$ is the valence of $\mathrm{Al}^{3+}$ ions. From Eqs. (13), (22), and (23), the volumetric strain in the oxide is

$$
\varepsilon=\frac{v_{\exp a n}-v_{\text {space }}}{v_{m / o}}=\left(\frac{\varepsilon_{V}}{1+\varepsilon_{V}}\right)-\beta \frac{x V_{A l}}{V_{o x}} .
$$

For the case of zero-strain $\varepsilon=0$,

$$
\beta=\left(\frac{\varepsilon_{V}}{1+\varepsilon_{V}}\right) \frac{V_{o x}}{x V_{A l}}=\frac{\varepsilon_{V}}{x} .
$$

The second step in Eq. (25) is due to $V_{o x}=V_{A 1} \times\left(1+\varepsilon_{V}\right)$, and $x=2$ is the valence of $\mathrm{O}^{2-}$ ions. According to experiments, ${ }^{38}$ the Pilling-Bedworth ratio $\left(1+\varepsilon_{V}\right)$ due to $\mathrm{Al} \rightarrow$ $\mathrm{Al}_{x} \mathrm{O}_{y}$ is 1.2 to 1.9 depending on electrolyte type, thus Eq. (25) gives $\beta=0.1$ to 0.45 . At the $\mathrm{m} / \mathrm{o}$ interface, as stated before, the relative rates of reactions in Eqs. (2) and (3) can be affected by $\mathrm{Al}$ substrate orientation, and so $\beta$ will change from the zero-strain value of $\varepsilon_{V} / x$ depending on the substrate orientation. On the one hand, the molar quantity of $\mathrm{O}^{2-}$ ions incorporated into the newly formed oxide per unit $\mathrm{m} / \mathrm{o}$ interface area per unit time by Eq. (3) equals $\left.j_{O, o x}\right|_{m / o} /(x \mathrm{~F})$, and this amount of $\mathrm{O}^{2-}$ ions binds with $\left.j_{O \text {,ox }}\right|_{m / O} /(y F)$ mole of $\mathrm{Al}^{3+}$ ions ionized from metal. On the other hand, Eq. (2) depletes $\left.j_{A l, o x}\right|_{m / o} /(y F)$ mole of $\mathrm{Al}^{3+}$ ions from the newly formed oxide at $\mathrm{m} / \mathrm{o}$ interface, which migrate towards the o/e interface for getting lost into the electrolyte. Thus, the oxygen content in the newly formed oxide is

$$
\begin{aligned}
O_{\text {content }} & =\frac{\left.j_{O, o x}\right|_{m / o} /(x F)}{\left.j_{O, o x}\right|_{m / o} /(x F)+\left.j_{O, o x}\right|_{m / o} /(y F)-\left.j_{A l, o x}\right|_{m / o} /(y F)} \\
& =\frac{y}{x(1-\beta)+y} .
\end{aligned}
$$



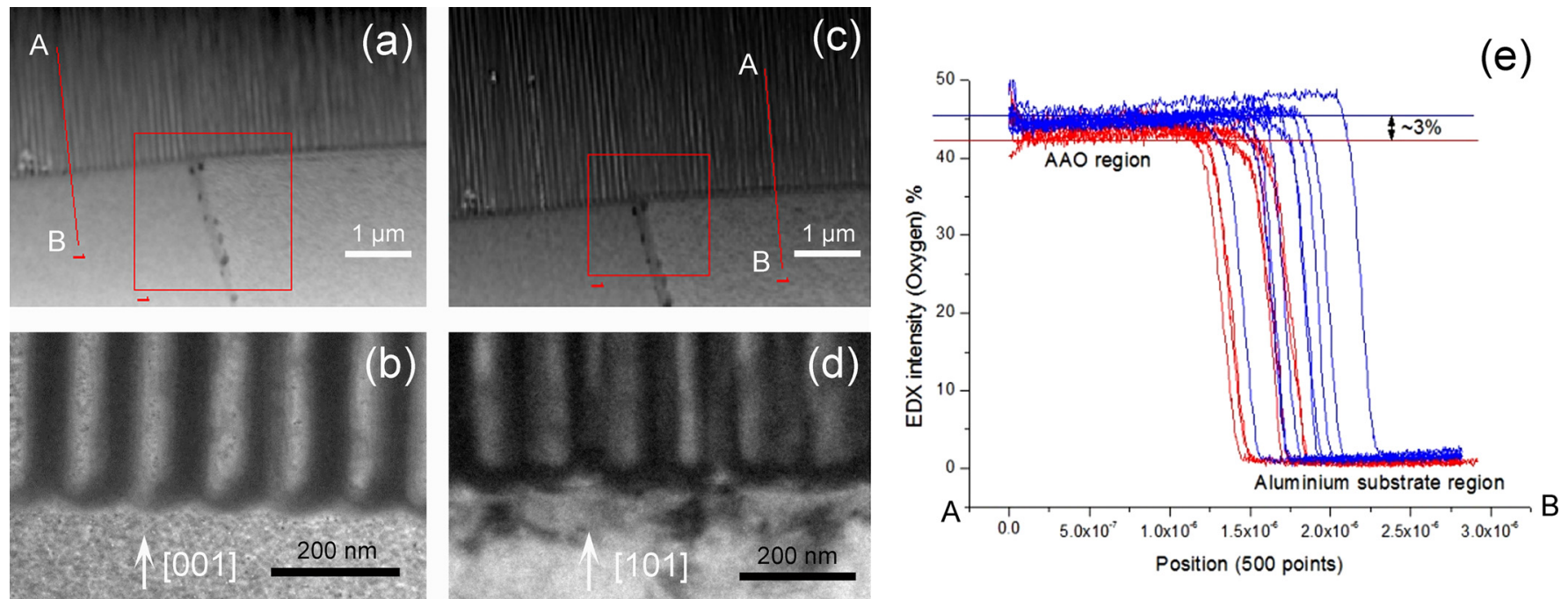

FIG. 5. EDX line profile measurements (from A to B with 500 profile points and dwell time $1200 \mu \mathrm{s}$ ) on (a) self-ordered and (c) disordered anodic porous alumina formed on (001) and (101) oriented Al grains. (b) and (d) are TEM images of the pore channels at the metal/oxide (m/o) interface on (001) and (101) Al grains, respectively. (e) EDX results of oxygen intensity in anodic porous alumina. The blue and red curves correspond to self-ordered and disordered anodic porous alumina, respectively. The anodization is conducted in $40 \mathrm{~V}, 0.3 \mathrm{M} \mathrm{H}_{2} \mathrm{C}_{2} \mathrm{O}_{4}, 17^{\circ} \mathrm{C}, 10 \mathrm{~h} 1$ st step, and $10 \mathrm{~h} 2 \mathrm{nd}$ step.

For two Al grains with different orientations, a difference of $\Delta \beta$ exists between them. From Eq. (26), the porous alumina grown on them would have a difference in oxygen content given by

$$
\Delta O_{\text {content }} \approx \frac{x y}{[x(1-\beta)+y]^{2}} \Delta \beta
$$

Therefore, a higher $\beta$ value, which would lead to stable pore channel growth and in-plane self-ordering in porous alumina according to Sec. IV, should be associated with higher oxygen content in the oxide according to Eq. (27). Physically, from Eq. (12), $\beta$ represents the ratio of the reaction rates of the ionization reaction, Eq. (2), to the oxidation reaction, Eq. (3), thus, a higher $\beta$ means more Al ionized and migrated away from the $\mathrm{m} / \mathrm{o}$ interface rather than being oxidized. As more ionized $\mathrm{Al}^{3+}$ ions are lost, the oxygen content in the residual oxide will increase, hence a higher $\beta$ corresponds to a higher $\mathrm{O}: \mathrm{Al}$ ratio in the oxide.

\section{B. Experimental verification}

To verify whether better self-ordering anodic porous alumina contains a higher oxygen content, the oxygen content was determined from the cross-section TEM samples by energy dispersive $\mathrm{x}$-ray spectroscopy (EDX) performed in a Philips Tecnai G2 20 S-TWIN Scanning Transmission Electron Microscope. Figs. 5(a) and 5(c) illustrate the TEM images, as well as typical line-profile measurements of the EDX tests performed on self-ordered and disordered porous alumina grown on two adjoining (001) and (101) Al grains, respectively. The framed regions in Figs. 5(a) and 5(c) were for the drift-correction during the data acquisition. The EDX line-profile measurements were made from the oxide region to the $\mathrm{Al}$ substrate region crossing the $\mathrm{m} / \mathrm{o}$ interface along the pore channel direction. Figs. 5(b) and 5(d) show the bottom of the pore channels at the m/o interface for self-ordered and disordered porous alumina on (001) and (101) Al grains, respectively. The scalloped shape of the oxide barrier layer at the pores' bottom can be clearly observed. The EDX results shown in Fig. 5(e) clearly show that the self-ordered alumina grown on (001) Al contains typically 3\% more oxygen than the disordered alumina grown on (101) Al. The different curves in Fig. 5(e) are repeated measurements at different locations over a wide length of the m/o interface on both sides of the (001)/(101) Al grain boundary separately, so that this oxygen composition difference is rather reliable. In Eq. (27), a typical value for $\beta$ is $3 / 7$ according to experiments, ${ }^{25}$ and thus $\Delta O_{\text {content }} \approx 0.35 \times \Delta \beta$. If $\Delta \beta \approx 0.1$, which is the magnitude involved in the simulations in Figs. 3 and 4 , then $\Delta O_{\text {content }} \approx 3.5 \%$. This order of magnitude of oxygen content change is in good agreement with the EDX results in Fig. 5(e) between the self-ordered and disordered porous alumina.

\section{CONCLUSIONS}

The effects of substrate orientation on the in-plane selfordering qualities of anodic porous alumina are theoretically and experimentally investigated. Under the same anodization conditions, self-ordered porous alumina is formed on (001) oriented $\mathrm{Al}$ grains, whereas disordered porous pattern is formed on (101) Al grains. From TEM cross-section observation, the above difference of the in-plane self-ordering is due to the stable and unstable pore channel development in self-ordered and disordered porous alumina, respectively. EDX measurements revealed that the oxygen content in selfordered porous alumina is about $3 \%$ higher than that in the disordered counterpart. Numerical simulation of the pore channel growth during anodization was carried out based on a kinetics model in which the potential barrier at the oxide/ electrolyte interface is assumed to be the rate-determining step, while the substrate orientation affects the relative rates of the ionization and oxidation reactions of $\mathrm{Al}$ substrate at the metal/oxide interface. The simulated results show that stable pore channel growth in the porous alumina is 
associated with a higher ratio of the ionization to the oxidation reaction rates at the $\mathrm{m} / \mathrm{o}$ interface, and such a change in this ratio should correspond to $\sim 3.5 \%$ change in the oxygen content in the oxide, which is in good agreement with EDX experiments.

\section{ACKNOWLEDGMENTS}

The work described in this paper was supported by funding from the Research Grants Council (Project No. 7159/10E) and the University Grants Committee (Project No. SEGHKU06) of the Hong Kong Special Administrative Region, as well as from the Kingboard Professorship Endowment and the Hung Hing Ying Distinguished Professorship Scheme.

${ }^{1}$ G. E. Thompson and G. C. Wood, Nature 290, 230 (1981).

${ }^{2}$ H. Masuda and K. Fukuda, Science 268, 1466 (1995).

${ }^{3}$ W. Lee, R. Ji, U. Gösele, and K. Nielsch, Nature Mater. 5, 741 (2006).

${ }^{4}$ Z. Su and W. Zhou, Adv. Mater. 20, 3663 (2008).

${ }^{5}$ C. K. Y. Ng and A. H. W. Ngan, Chem. Mater. 23, 5264 (2011).

${ }^{6}$ H. Masuda, M. Yamada, F. Matsumoto, S. Yokoyama, S. Mashiko, M. Nakao, and K. Nishio, Adv. Mater. 18, 213 (2006).

${ }^{7}$ W. B. Choi, J. U. Chu, K. S. Jeong, E. J. Bae, J. W. Lee, J. J. Kim, and J. O. Lee, Appl. Phys. Lett. 79, 3696 (2001).

${ }^{8}$ W. Lee, H. Han, A. Lotnyk, M. A. Schubert, S. Senz, M. Alexe, D. Hesse, S. Baik, and U. Gösele, Nat. Nanotechnol. 3, 402 (2008).

${ }^{9}$ F. Matsumoto, K. Nishio, and H. Masuda, Adv. Mater. 16, 2105 (2004).

${ }^{10}$ A. P. Li, F. Müller, A. Birner, K. Nielsch, and U. Gösele, J. Appl. Phys. 84, 6023 (1998).

${ }^{11}$ H. Masuda, F. Hasegwa, and S. Ono, J. Electrochem. Soc. 144, L127 (1997).

${ }^{12}$ K. Nielsch, J. Choi, K. Schwim, R. B. Wehrspohn, and U. Gösele, Nano Lett. 2, 677 (2002).
${ }^{13}$ K. Y. Ng, Y. Lin, and A. H. W. Ngan, Acta Mater. 57, 2710 (2009).

${ }^{14}$ K. Y. Ng and A. H. W. Ngan, Scr. Mater. 66, 439 (2012).

${ }^{15}$ G. Beck and R. Bretzler, Mater. Chem. Phys. 128, 383 (2011).

${ }^{16}$ G. Beck and K. Retrikowski, Surf. Coat. Technol. 202, 5084 (2008).

${ }^{17}$ K. S. Napolskii, I. V. Roslyakov, A. Y. Romanchuk, O. O. Kapitanova, A. S. Mankevich, V. A. Lebedev, and A. A. Eliseev, J. Mater. Chem. 22, 11922 (2012).

${ }^{18}$ C. Cheng and A. H. W. Ngan, Electrochim. Acta 56, 9998 (2011).

${ }^{19}$ C. Cheng and A. H. W. Ngan, Nanotechnology 24, 215602 (2013).

${ }^{20} \mathrm{~W}$. Rasband, IMAGEJ, release 1.44 (NIH, USA, 2011), http://rsb.info.nih. gov/ij/.

${ }^{21}$ V. P. Parkhutik and V. I. Shershulsky, J. Phys. D: Appl. Phys. 25, 1258 (1992).

${ }^{22}$ G. K. Singh, A. A. Golovin, and I. S. Aranson, Phys. Rev. B 73, 205422 (2006).

${ }^{23}$ C. Cherki and J. Siejka, J. Electrochem. Soc. 120, 784 (1973).

${ }^{24}$ M. M. Lohrengel, Mater. Sci. Eng. R. 11, 243 (1993).

${ }^{25}$ J. Siejka and C. Ortega, J. Electrochem. Soc. 124, 883 (1977).

${ }^{26}$ J. P. O'Sullivan and G. C. Wood, Proc. R. Soc. London, Ser. A 317, 511 (1970).

${ }^{27}$ J. E. Houser and K. R. Hebert, Nature Mater. 8, 415 (2009).

${ }^{28}$ F. Li, L. Zhang, and R. M. Metzger, Chem. Mater. 10, 2470 (1998).

${ }^{29}$ N. Cabrera and N. F. Mott, Rep. Prog. Phys. 12, 163 (1949).

${ }^{30}$ N. Q. Zhao, X. X. Jiang, C. S. Shi, J. J. Li, Z. G. Zhao, and X. W. Du, J. Mater. Sci. 42, 3878 (2007)

${ }^{31}$ S. Ono, M. Saito, M. Ishiguro, and H. Asoh, J. Electrochem. Soc. 151, B473 (2004).

${ }^{32}$ C. Cheng, K. Y. Ng, and A. H. W. Ngan, AIP Adv. 1, 042113 (2011).

${ }^{33}$ A. L. Friedman, D. Brittain, and L. Menon, J. Chem. Phys. 127, 154717 (2007).

${ }^{34}$ J. W. Diggle, T. C. Downie, and C. W. Goulding, Chem. Rev. 69, 365 (1969).

${ }^{35}$ T. Valand and K. E. Heusler, J. Electroanal. Chem. 149, 71 (1983).

${ }^{36}$ MATLAB, R2009a, version 7.8.0.347, The Mathworks Inc., 2009.

${ }^{37}$ R. E. Smallman and A. H. W. Ngan, Physical Metallurgy and Advanced Materials (Elsevier, Amsterdam, 2007).

${ }^{38}$ L. Arurault, Trans. Inst. Met. Finish. 86, 51 (2008). 